\title{
Avaliação da saúde bucal de pacientes cirróticos em fila de transplante hepático
}

\author{
Assessment of oral health of cirrhotic patients in liver transplant waiting list. \\ Evaluación de la salud bucal de pacientes cirróticos en la lista de espera de trasplante hepático.
}

\author{
Karina Pidhorodeckyj 1,a, Jefferson da Rocha Tenório ${ }^{2, b}$, Nathália Tuany Duarte ${ }^{2, b}$, Bruna Di Profio ${ }^{1, c}$,

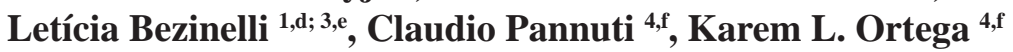

\section{RESUMO}

Objetivo: Avaliar a saúde bucal de pacientes com cirrose hepática em fila de transplante. Materiais e Método: Foram avaliados 103 pacientes cirróticos quanto às características sócio-demográficas e histórico médico da cirrose hepática (etiologia, tempo de inclusão na fila de transplante, valor do MELD - Model for End-Stage Liver Disease, complicações da cirrose e medicações em uso). A avaliação da saúde oral foi feita através do índice de higiene oral simplificado (IHO-S), do índice de cárie dental (CPOD) e da sialometria. Foram realizadas análises descritivas após a tabulação dos dados. Resultados: A população estudada apresentava idade média de 54 anos, o sexo masculino era o mais prevalente e apenas $44 \%$ concluíram o ensino fundamental. Hepatite C e cirrose alcoólica foram as etiologias que, com mais frequência, levaram os pacientes a indicação de transplante hepático. Varizes esofágicas, ascite e hipertensão portal foram as complicações mais frequentes. O CPOD médio da população estudada foi considerado alto $(23,3)$. Conclusão: A saúde bucal de pacientes cirróticos em fila de transplante não é satisfatória. Estudos longitudinais são necessários para investigar a relação entre focos de infecção oral e a ocorrência de infecções no pós-transplante hepático.

DESCRITORES: Cirrose hepática, saúde bucal, infecção focal dentária, hepatite, transplante de órgãos, transplante hepático, imunossupressão.

Faculdade de Odontologia, Universidade de São Paulo. São Paulo, Brazil.

2 Doutorado em Ciências Odontológicas (Patologia Oral e Maxilofacial e Pacientes Especiais), Faculdade de Odontologia, Universidade de São Paulo. São Paulo, Brazil.

3 Hospital Israelita Albert Einstein. São Paulo, Brazil.

4 Disciplina de Patologia Oral e Maxilofacial, Departamento de Estomatologia, Faculdade de Odontologia, Universidade de São Paulo. São Paulo, Brazil.

a Cirurgiã dentista; ' ${ }^{\text {Aluno; }}{ }^{\circ}$ Mestre em Ciências Odontológicas (Periodontia); ${ }^{a}$ Mestre e Doutora em Ciências Odontológicas; e Cirurgiã dentista; ${ }^{\dagger}$ Professora 


\section{SUMMARY}

Objectives: To assess the oral health of patients waiting for a liver transplantation. Material and Methods: A total of 103 cirrhotic patients were evaluated for socio-demographic characteristics and medical history of liver cirrhosis (etiology, time of inclusion in the transplant list, MELD - Model for End Liver Disease - value, complications of cirrhosis and routine medications in use). In addition, simplified oral hygiene index (IHO-S), dental caries index (DMFT) and sialometry were performed to verify oral health. After tabulation of the data, descriptive analyzes were performed. Results: The mean age was 54 years, the male gender was the most prevalent and the elementary school was completed by only $44 \%$ of the studied population. Hepatitis $C$ and alcoholic cirrhosis were the etiologies that, more frequently, led the patients to indication for liver transplantation. Esophageal varices, ascites and portal hypertension were the complications of cirrhosis most observed. The mean DMFT of the studied population was considered high (23.3 ). Conclusions: The oral health of cirrhotic patients in the waiting list for transplantation is not satisfactory. Longitudinal studies are needed to investigate the relationship between foci of oral infections and the occurrence of infections post-transplant liver.

KEYWORDS: Liver cirrhosis, oral health, dental focal infection, hepatitis, organ transplantation, liver transplantation, immunosuppression

\section{RESUMEN}

Objetivo: Evaluar la salud bucal de pacientes con cirrosis hepática en fila de trasplante. Material y Métodos: Fueron evaluados 103 pacientes cirróticos en cuanto a las características sociodemográficas e historial médico de cirrosis hepática (etiología, tiempo de inclusión en fila de trasplante, valor del MELD - Model for End-Stage Liver Disease, complicaciones de la cirrosis y medicaciones en uso). Así mismo fueron realizados los siguientes índices para evaluación de la salud bucal: índice de higiene oral simplificado (IHO-S), índice de caries dental (CPOD) y sialometría. Fueron realizados análisis descriptivos después de la tabulación de datos. Resultados: La población estudiada presentaba edad media de 54 años, el sexo masculino era el más prevalente y sólo el 44\% concluyeran la educacion fundamental. La hepatitis C y la cirrosis alcohólica fueron las etiologias que con más frecuencia, llevaron a los pacientes a indicación de trasplante hepático. Las complicaciones de la cirrosis más observadas fueron várices esofágicas, ascitis e hipertensión portal. El CPOD medio de la población estudiada fue considerado alto, comparado con personas normoreactivas del mismo grupo etáreo (23,3 y 16,75 respectivamente ). Conclusiones: La salud bucal de pacientes cirroticos en fila de trasplante no es satisfactoria. Estudios longitudinales son necesarios para investigar la relación entre focos de infección bucal y la ocurrencia de infecciones en el post transplante hepático.

PALABRAS CLAVE: Cirrosis hepática, salud bucal, infección focal dentária, hepatitis, trasplante de órganos, trasplante hepático, inmunosupresión.

\section{INTRODUÇÃO}

Pacientes cirróticos em fila de transplante apresentam alterações na resposta imune inata e adaptativa, desenvolvendo um estado de imunossupressão adquirida (1). Essa característica aumenta o risco de doenças infecciosas nesses pacientes, incluindo doença periodontal (2-5). Por sua vez, pacientes transplantados hepáticos são submetidos à imunossupressão terapêutica, que também pode levar ao aparecimento de importantes doenças infecciosas, sendo a boca uma possível fonte dessas doenças. Patógenos e toxinas advindos de infecções dentais e periodontais são capazes de se propagar pela corrente sanguínea, causando alterações sistêmicas que podem aumentar o risco para doenças cardiovasculares, respiratórias, doença renal crônica e até diabetes mellitus, embora a relação causa-efeito não tenha sido totalmente elucidada $(6,7)$. 
Diversas equipes médicas subordinam o transplante, seja de medula ou órgãos sólidos, à erradicação de focos infecciosos, sendo a adequação da saúde oral um fator condicionante. A avaliação e o tratamento odontológico prévios já são bem estabelecidos para pacientes na fila de transplante de medula e de órgãos sólidos como rim e pulmão, uma vez que qualquer tipo de bacteremia após o transplante é capaz de reduzir em até um ano a taxa de sobrevida global do paciente (8-13). As infecções apresentam substancial impacto na progressão da falha hepática, na ocorrência de complicações relacionadas com a imunossupressão pós-transplante hepático e nas elevadas taxas de morbidade e mortalidade associadas com o transplante hepático (14). Assim, a necessidade do tratamento odontológico justifica-se tanto na fase pré quanto na fase pós-transplante desse tipo de paciente.

O paciente na fila de transplante de fígado pode apresentar diversas alterações orais que devem ser avaliadas e manejadas pelo dentista. Dentre elas salientam-se o sangramento gengival espontâneo e as deficiências nutricionais capazes de predispor os mesmos a manifestações orais como a queilite angular, glossite, petéquias e equimoses (15). A sobrevida após o transplante hepático tem aumentado sobremaneira em decorrência da melhora nos métodos de transporte dos órgãos, maior compreensão a respeito do sistema imunológico do paciente e do desenvolvimento de novas drogas imunossupressoras (16-18). A odontologia também tem contribuído para melhora na sobrevida desses pacientes ao eliminar possíveis focos de infecção. Por outro lado, o aumento da expectativa de vida desses pacientes demanda um aumento dos cuidados globais de saúde, entre eles o tratamento odontológico.

Apesar de o fígado ser o segundo órgão de maior demanda para transplante no Brasil e no mundo (19), pouco se sabe a respeito da saúde bucal desses pacientes. A necessidade da avaliação odontológica prévia ao transplante não é protocolo em todas as equipes médicas, visto que poucos trabalhos conseguiram de fato associar infecções pós transplante a focos de origem bucal $(20,21)$. Aberg e colaboradores em 2014 avaliaram 116 pacientes cirróticos e foi observado que o Streptococcus viridans, um importante e frequente patógeno bucal, estava presente dentre as bactérias encontradas no líquido ascítico de pacientes com peritonite bacteriana. Mas isso ocorreu apenas nos pacientes que tinham múltiplas infecções dentais, permitindo supor que as infecções dentais são capazes de influenciar o curso clínico da doença hepática de pacientes cirróticos (22).

Entretanto, a eliminação de focos infecciosos de origem dental tem sido uma das preocupações de poucos centros transplantadores de fígado (23). Talvez porque poucos centros conheçam a qualidade da saúde bucal de seus pacientes em fila de transplante hepático.

Este trabalho tem como objetivo descrever as características da saúde bucal dos pacientes previamente ao transplante de fígado, afim de, posteriormente, desenvolver um protocolo de atenção bucal para tais pacientes.

\section{MATERIAIS E MÉTODO}

O Comitê de Ética em Pesquisa (CEP) da Faculdade de Odontologia da Universidade de São Paulo (FOUSP) aprovou o estudo, sendo que o Termo de Consentimento Livre e Esclarecido (TCLE) foi preenchido por todos os pacientes que participaram da pesquisa.

O grupo de estudo foi constituído por todos os pacientes cirróticos que estavam em fila de transplante hepático e que foram atendidos no Centro de Atendimento a Pacientes Especiais (CAPE) da Faculdade de Odontologia da Universidade de São Paulo (FOUSP) entre Março de 2009 e Novembro de 2012.

Os pacientes foram submetidos a exame clínico completo, constando de anamnese e exame físico intra-oral. Foram colhidas informações referentes às características sócio-demográficas (sexo, idade, cor da pele, grau de escolaridade), informações sobre a frequência de higienização oral e sobre a história médica referente à cirrose (etiologia da cirrose, tempo de inclusão na fila de transplante, valor do MELD Model of End-Stage Liver Disease, complicações da cirrose, e uso de medicações de rotina).

O exame físico intra-oral foi realizado em ambiente ambulatorial, com espelho bucal e luz artificial por dois examinadores previamente calibrados. $\mathrm{O}$ índice de higiene oral simplificado (IHO-S), índice de cárie dental (CPOD) e sialometria também foram realizados. 
Para a execução do IHO-S utiliza-se de um evidenciador de placa (Replak ${ }^{\circledR}$, Dentsply) com o objetivo e identificar e quantificar o biofilme e cálculo dental nas superfícies dentárias. Essa avaliação considera, usualmente, as seguintes superfícies dentais: faces vestibulares dos primeiros molares superiores, um incisivo central superior e um incisivo central inferior, além das faces linguais dos primeiros molares inferiores. Após a aplicação do revelador e seleção das faces, são atribuídos os seguintes escores: Quanto ao biofilme: 0 - Ausência de biofilme, 1 - Presença de biofilme apenas no terço cervical, 2 - Biofilme presente até o terço médio, 3 - Biofilme recobre mais de 2/3 da superfície dentária; Quanto à presença de cálculo: 0 - Ausência de cálculo dental, 1 - Presença de cálculo dental supra-gengival apenas no terço cervical, 2 - cálculo dental supra-gengival presente até o terço médio, 3 - Cálculo dental supra-gengival recobre mais de 2/3 da superfície dental. Com a somatória do índice de biofilme e de cálculo dental obtém-se o índice de higiene oral (24).

O índice CPOD identifica: dentes permanentes cariados, perdidos e obturados, com o objetivo de estimar a experiência de cárie dental na dentição permanente. O diagnóstico foi feito respeitando os critérios da OMS (25).

Os pacientes também foram submetidos a exame para avaliar o fluxo salivar conforme metodologia descrita a seguir: a) A saliva foi coletadaduas horas após a última refeição notificada pelo paciente; b) Foi solicitado ao paciente que mastigasse um pequeno pedaço de cera parafina por 30 segundos e que a saliva, acumulada em sua boca, fosse deglutida; c) Após isso, o paciente deveria mastigar a cera por mais 2 minutos, sem deglutir a saliva acumulada, e esta, por sua vez,deveria ser depositada em um recipiente. Essa etapa foi repetida duas vezes; d) A medição da quantidade de saliva estimulada foi feita mediante o uso de uma seringa hipodérmica; e) Foram considerados valores indicativos de hipossalivação os resultados menores que $0,7 \mathrm{~mL} / \mathrm{min}$.

A saúde oral foi avaliada através do cálculo do CPOD e IHO-S. . Foi realizada a análise descritiva dos dados, sendo calculada a frequência das variáveis, média e desvio padrão com auxílio do programa estatístico Statistical Program for the Social Sciences (SPSS 20.0).

\section{RESULTADOS}

Durante o período de estudo, foram colhidos dados de 103 pacientes quanto às características demográficas e história médica. A maioria dos pacientes era do sexo masculino ( $n=75 / 72,8 \%)$, leucoderma $(n=86 / 83,5 \%)$ e com média de idade de 54 anos. Além disso, dentre oitenta e quatro pacientes que declararam o grau de escolaridade, a maioria apresentava apenas o ensino fundamental completo ( $\mathrm{n}=37 / 44 \%)$.

Quanto a história médica referente à hepatopatia crônica dos pacientes, a hepatite C (45,6\%) e o consumo de álcool $(23,3 \%)$ foram os principais fatores etiológicos da cirrose hepática. Também foi constatado que cada paciente apresentou mais de uma complicação da cirrose, sendo as varizes esofágicas $(57,3 \%)$, ascite $(52,4 \% \%)$ e hipertensão portal $(47,6 \%)$ as mais frequentes. Icterícia $(44,7 \%)$ e fadiga $(41,7 \%)$ foram os sinais e sintomas mais observados. Também foi possível investigar a presença de outras comorbidades sistêmicas sendo a anemia (30,1\%), o diabetes $(28,2 \%)$ e a hipertensão arterial sistêmica $(15,5 \%)$ as mais relatadas. Os pacientes faziam uso de mais de uma medicação de rotina, em que os anti-hipertensivos, diuréticos e gastroprotetores foram os mais frequentemente utilizados (tabela 1).

Todos os pacientes deste estudo buscaram tratamento odontológico para adequação da saúde oral enquanto estavam na fila de transplante hepático e apresentaram, em média, um MELD de 17 pontos e um tempo médio superior a 6 meses na fila de transplante. Após anamnese e coleta de dados sobre o histórico da doença hepática, setenta e seis pacientes permitiram o exame clínico para avaliação do CPOD e quarenta e seis admitiram a aplicação do evidenciador de placa para verificação do IHO-S e exame do fluxo salivar. Os valores mínimo, máximo, média e desvio padrão para cada índice investigado estão dispostos na Tabela 2. Sendo assim, o CPOD médio da população estudada foi de 23,3. Somado a esses dados, setenta e seis pacientes foram questionados quanto à frequência de higienização oral, o que permitiu identificar que a maior parte dos pacientes ( $\mathrm{n}=44 / 57,9 \%$ ) higieniza a cavidade oral, em média, três vezes ao dia. 
Tabela 1. História Médica da Doença Atual.

\begin{tabular}{|c|c|c|}
\hline Etiologia da Doença Hepática & $\mathbf{n}$ & $\%$ \\
\hline Hepatite C & 47 & 45,6 \\
\hline Cirrose alcoólica & 24 & 23,3 \\
\hline Cirrose Criptogênica & 15 & 14,6 \\
\hline Hepatite B & 10 & 9,7 \\
\hline Cirrose biliar primária & 3 & 2,9 \\
\hline Outras & 4 & 3,9 \\
\hline \multicolumn{3}{|l|}{ Complicações da cirrose* } \\
\hline Varizes Esofágicas & 59 & 57,3 \\
\hline Ascite & 54 & 52,4 \\
\hline Hipertensão Portal & 49 & 47,6 \\
\hline Encefalopatia & 47 & 45,6 \\
\hline Coagulopatia & 42 & 40,8 \\
\hline Plaquetopenia & 34 & 33,0 \\
\hline Hiperesplenismo & 29 & 28,1 \\
\hline Hepatocarcinoma & 11 & 10,7 \\
\hline Outras & 10 & 9,7 \\
\hline \multicolumn{3}{|l|}{ Sinais e sintomas relatados* } \\
\hline Icterícia & 46 & 44,7 \\
\hline Fadiga & 43 & 41,7 \\
\hline Perda de peso & 34 & 33 \\
\hline Prurido & 17 & 16,5 \\
\hline Episódio hemorrágico** & 34 & 33 \\
\hline Edema & 26 & 25,2 \\
\hline Náuseas & 22 & 21,4 \\
\hline Sangramento gengival & 18 & 17,5 \\
\hline Vômito & 10 & 9,7 \\
\hline \multicolumn{3}{|l|}{ Comorbidades* } \\
\hline Hipertensão Arterial Sistêmica & 16 & 15,5 \\
\hline Síndrome Hepato-renal & 12 & 11,7 \\
\hline Diabetes & 29 & 28,2 \\
\hline Desordens Pulmonares & 10 & 9,7 \\
\hline Cardiopatias & 12 & 11,7 \\
\hline Anemia & 31 & 30,1 \\
\hline \multicolumn{3}{|l|}{ Medicações de Rotina* } \\
\hline Anti-hipertensivos & 58 & 56,3 \\
\hline Diuréticos & 57 & 55,3 \\
\hline Gastroprotetores & 46 & 44,7 \\
\hline Lactulose & 28 & 27,2 \\
\hline Vitaminas & 25 & 24,3 \\
\hline Antibióticos & 13 & 12,6 \\
\hline Insulina & 12 & 11,7 \\
\hline Cardiotônicos & 12 & 11,7 \\
\hline
\end{tabular}

* O mesmo paciente poderia apresentar mais de uma condição **Episódio hemorrágico não gengival 
Tabela 2. Valores do CPOD, IHO-S e Fluxo Salivar

\begin{tabular}{ccccc}
\hline & Mínimo & Máximo & Média & DP* \\
\hline CPOD (n = 76) & 5 & 32 & 23,3 & 6,61 \\
Cariados & 0 & 14 & 3,47 & 3,47 \\
Perdidos & 0 & 32 & 12,77 & 8,73 \\
Obturados & 0 & 21 & 7,22 & 5,51 \\
IHO-S (n = 46) & 0 & 6 & 2,05 & 1,19 \\
Placa & 0 & 3 & 1,50 & 0,81 \\
Cálculo & 0 & 1,5 & 0,54 & 0,55 \\
Fluxo Salivar (n=46) & 0,3 & 2,12 & 1,17 & 0,49 \\
\hline
\end{tabular}

* Desvio Padrão

\section{DISCUSSÃO}

Pacientes com cirrose hepática na fila de transplante devem passar por consulta e execução de tratamento odontológico previamente ao transplante hepático para evitar complicações pós-transplante capazes de influenciar na perda do enxerto. Os dados demográficos descritivos sobre idade dos pacientes em programação de transplante nesse trabalho corroboram com informações da literatura. Kim e colaboradores em 2015 relataram na Annual Data Report que a maioria dos pacientes submetidos a transplante hepático em 2013 nos Estados Unidos era do sexo masculino (62,7\%), similarmente, $72.8 \%$ da nossa amostra foi composta por homens (26).

As causas que mais frequentemente levam pacientes adultos a desenvolver cirrose hepática são as hepatites virais e o abuso de álcool. O vírus da hepatite C, em 2013, foi o motivo pelo qual 29,4\% dos pacientes hepatopatas dos EUA aguardavam um transplante hepático, enquanto 24,1\% entraram na fila de espera devido à cirrose alcoólica. De forma semelhante, mais da metade dos pacientes desse estudo (78,6\%) tiveram como causa primária hepatites virais (B e C) e cirrose relacionada ao álcool. Aqui se ressalta a importância de políticas públicas de saúde e programas sociais que conscientizem a população quanto às formas de transmissão e prevenção das hepatites virais, bem como o combate à dependência alcoólica.

No presente estudo, o CPOD médio foi de 23,3 que é considerado uma prevalência alta da experiência de cárie, segundo a OMS, quando considerado a média da faixa etária da população estudada (25). Conforme dados da mais recente Pesquisa Nacional de Saúde Bucal realizada em 2010, o CPOD médio da população brasileira com idade entre 35 e 44 anos foi de 16,75 (27). Infere-se que as possíveis causas para que esse CPOD esteja elevado na pesquisa em questão sejam: 1) as questões socioeconômicas envolvidas, uma vez que a maior parte dos pacientes apresentou reduzido grau de escolaridade, o que pode explicar o baixo acesso à informação sobre cuidados orais, 2) a negligência ao atendimento de pacientes com cirrose hepática, devido ao comprometimento sistêmico que eles apresentam e à falta de conhecimento sobre a fisiopatologia da doença por parte dos cirurgiões-dentistas e 3) o uso de diversas medicações que possuem como efeito colateral a redução do fluxo salivar que, consequentemente, predispõe ao aumento de cárie.

A saliva contém enzimas, entre elas a lactoferrina e a lisozima, que são capazes de degradar a parede de peptidoglicana de bactérias como o Streptococcus spp. e o Staphylococcus spp. Essas funções, em conjunto, atuam no combate a estes patógenos e na diminuição da quantidade de cáries (28). Contudo, é bem conhecido o fato de que algumas medicações podem resultar na sensação de boca seca (xerostomia) e, por vezes, em hipossalivação. No presente estudo, grande parte dos pacientes fazia uso de um ou mais medicamentos de rotina, entre eles anti-hipertensivos e diuréticos. Esses medicamentos podem estar relacionados tanto com hipossalivação quanto com a xerostomia (29). Esse achado pode justificar o elevado índice de cárie nesses pacientes.

Guggenheimer e colaboradores reportaram a ausência 
de estudos que tenham avaliado a condição de saúde oral em pacientes adultos com cirrose hepática na fila de transplante e em pacientes já transplantados (30). O presente estudo apresenta algumas limitações. Dentre elas destacam-se o tamanho da amostra e a falta de aceitação dos pacientes em se submeter à verificação do CPOD, IHOS e exame do fluxo salivar.

\section{CONCLUSÃO}

A saúde bucal de pacientes cirróticos em fila de transplante não é satisfatória. Estudos longitudinais são necessários para investigar a relação entre focos de infecção oral e a ocorrência de infecções no pós-transplante hepático.

\section{Correspondência:}

Karem L. Ortega

Correo electrónico: klortega@usp.br

\section{REFERÊNCIAS}

1. Albillos A, Lario M, Álvarez-Mon M. Cirrhosis-associated immune dysfunction: distinctie features and clinical relevance. J Hepatol. 2014; 61(6): 1385-1396.

2. Guggenheimer J, Eghtesad B, Close JM, Shay C, Fung JJ. Dental health status of liver transplant candidates. Liver Transpl. 2007; 13(2):280-286.

3. Oettinger-Barak O, Barak S, Machtei EE, Ardekian L, Baruch Y, Peled M. Periodontal changes in liver cirrhosis and post-transplantation patients. I:clinical findings. J Periodontol. 2001; 72(9):1236-1240.

4. Oettinger-Barak O, Machtei EE, Barak S, Baruch Y, Ardekian L, Peled M. Periodontal changes in liver cirrhosis and post-transplantation patients. II:radiographic findings. J Periodontol. 2002; 73(3): 313-316.

5. Silva-Santos PS, Fernandes KS, Gallottini MH. Assessment and management of oral health in liver transplant candidates. J Appl Oral Sci. 2012; 20(2): 241-245

6. Pizzo G, Guiglia R, Lo-Russo L, Campisi G. Dentistry and internal medicine: from the focal infection theory to the periodontal medicine concept. Eur J Intern Med. 2010; 21 (6): 496-502.

7. Vasanthan A, Dallal N. Periodontal treatment considerations for cell transplant and organ transplant patients. Periodontol. 2000. 2007; 44:82-102.

8. Tinoco-Araujo JE, Orti-Raduan ES, Santos D, et al. Oral health-related quality of life before hematopoietic stem cell transplantation. Clin Oral Investig. 2015; 19(9):2345-2349.

9. Palmer SC, Ruospo M, Wong G, et al. Dental health and mortality in people with end-stage kidney disease treated with hemodialysis: A multinational cohort study. Am J Kidney Dis. 2015; 66(4):666-676.

10. Ruospo M, Palmer SC, Craig JC, et al. Prevalence and severity of oral disease in adults with chronic kidney disease: a systematic review of observational studies. Nephrol Dial Transplant. 2014; 29(2):364-375.

11. Marcinkowski A, Ziebolz D, Kleibrink BE, et al. Deficits in oral health behavior and oral health status in patients after lung transplantation. Clin Respir J. 2018;12(2):721-730. doi: 10.1111/crj.12585

12. Zwiech R, Bruzda-Zwiech A. Does oral health contribute to post-transplant complications in kidney allograft recipients? Acta Odontol Scand. 2013; 71(34):756-763. doi: 10.3109/00016357.2012.715203

13. Kim SI, Kim YJ, Jun YH, et al. Epidemiology and risk factors for bacteremia in 144 consecutive living-donor liver transplant recipients. Yonsei Med J. 2009; 50(1):112-121. doi: 10.3349/ymj.2009.50.1.112

14. Jalan R, Fernandez J, Wiest R, et al. Bacterial infections in cirrhosis: a positionstatement based on the EASL Special Conference 2013. J Hepatol. 2014; 60(6):1310-1324. doi: 10.1016/j.jhep.2014.01.024

15. Gallili D. A modern approach to prevention and treatment of oral bleeding in patients with hepatocellular disease. Oral Surg. 1982; 54(3): 277-280.

16. Morrissey PE, Madras PN, Monaco AP. History of solid organ transplantation. En: Kuo PC, Schroeder RA, Johnson LB, editors. Clinical management of the transplant patient. London: Arnold; 2001. p. 1-26.

17. Tilney NL. Transplantation and its biology: from fantasy to routine. J Appl Physiol. 2000; 89(5):1681-1689.

18. Glassman P, Wong C, Gish R. A review of liver transplantation for the dentist and guidelines for dental management. Spec Care Dent. 1993(2): 13: 74-80.

19. Associação Brasileira de Transplante de Órgãos. Registro Brasileiro de Transplantes. RBT. 2016; 22(1):0-0.

20. Wilson RL, Martinez-Tirado J, Whelchel J, Lordon RE. Occult dental infection in renal transplant patients. Am J Kidney Dis. 1982; 2(3):354-356.

21. Reyna J, Richardson JM, Mattox DE, Banowsky LH, Nicastro-Lutton JJ. Head and neck infection after renal transplantation. JAMA. 1982; 247(24):3337-3339.

22. Aberg F, Helenius-Hietala J, Meurman J, Isoniemi $\mathrm{H}$. Association between dental infections and the clinical course of chronic liver disease. Hepatol Res. 2014;44(3):349-53.

23. Helenius-Hietala J, Meurman JH, Höckerstedt K, Lindqvist C, Isoniemi H. Effect of the aetiology and severity of liver disease on oral health and dental treatment prior to transplantation. Transpl Int. 2012; 25(2):158-65

24. Greene JC, Vermillion JR. The simplified oral hygiene index. J Am Dent Assoc. 1964; 68: 7-13.

25. World Health Organization. Oral health surveys: basic methods. 5th ed. Geneva: WHO; 2013. 
26. Kim WR, Smith JM, Skeans MA, et al. OPTN/SRTR 2012 Annual Data Report: Liver. Am J Transplant. 2014; 1:69-96.

27. Brasil Ministério da Saúde; Secretaria de Atenção à Saúde; Departamento de atenção básica. Projeto SBBrasil 2010: Pesquisa Nacional de Saúde Bucal - Resultados Principais. Brasília: Ministério da Saúde;2014.

28. Tanasiewicz M, Hildebrandt T, Obersztyn I. Xerostomia of various etiologies: A review of the literature. Adv Clin Exp Med. 2016; 25(1):199-206.

29. Nonzee V, Manopatanakul S, Khovidhunkit SO. Xerostomia, hyposalivation and oral microbiota in patients using antihypertensive medications. J Med Assoc Thai. 2012; 95(1): 96-104.

30. Guggenheimer J, Mayher D, Eghtesad B. A survey of dental care protocols among US organ transplant centers. Clin Transplant. 2005; 19(1): 15-18.

Recibido: 09-06-2018

Aceptado: 28-10-2018 